\title{
Coherent Vortex Motion in Superconducting Nanobridges Based on YBaCuO Thin Films
}

\author{
M.V.Pedyash, G.J.Gerritsma, D.H.A.Blank and H.Rogalla \\ Department of Applied Physics, University of Twente, \\ P.O.Box 217, 7500 AE Enschede, the Netherlands
}

\begin{abstract}
Submicron bridges in superconducting $\mathrm{YBaCuO}$ thin films with typical linear dimensions of about $100 \mathrm{~nm}$ have been fabricated. Experimental current-voltage characteristics of these structures are in reasonable agreement with numerical simulations based on true two dimensional viscous vortex motion and indicate the effect of switching from a single path motion at low transport currents to multiple paths at higher currents. The value of the viscous drag coefficient of this vortex motion is found to be about $10^{-9} \mathrm{~kg} / \mathrm{m} \cdot \mathrm{sec}$, which is about two orders of magnitude lower than one estimated from the Bardeen-Stephen model. Critical current densities in the investigated nanobridges are up to $5 \cdot 10^{10} \mathrm{~A} / \mathrm{m}^{2}$ at $\mathrm{T}=4.2 \mathrm{~K}$. The critical current as a function of the width of the bridges indicates the dominating role of edge pinning.
\end{abstract}

\section{INTRODUCTION}

Up to date, the development of devices based on high $T_{c}$ superconductors shows that fabrication simplicity of such structures is the key to success. High $T_{c}$ Josephson junctions made by multilayer techniques are rather complicated in preparation and therefore have quite irreproducible characteristics. This fact raises an interest of using more simple superconducting structures like thin film submicron bridges [1],[2]. Superconducting bridges structured in thin films with dimensions less than a few times the coherence length $\xi$ show true Josephson behaviour [3]. Having negligible low capacitance they have the advantage of very high maximum operation frequency. Larger structures with dimensions up to the effective magnetic field penetration depth $\lambda_{\perp}$ also show some Josephson-like effects. The behaviour of such structures is determined by Abrikosov vortices motion [1],[4]. In the low temperature and low transport current regime this motion can be coherent due to the strong interaction between vortices, causing periodical oscillations in the electric and magnetic field emitted by the bridge [5]. By coupling of the periodic vortex motion with the external microwave irradiation Shapiro-like steps can be observed in the current-voltage (I-V) characteristics of these submicron bridges [4].

In this article we present experimental I-V characteristics of submicron bridges with typical linear sizes of about $100 \mathrm{~nm}$ prepared by combination of e-beam lithography and argon plasma milling and compare them with calculated I-V

Manuscript received October 17, 1994 curves, derived from computer simulations of viscous vortex motion inside superconducting submicron bridges of different shapes. Simulations have been performed following the method proposed by Aslamazov and Larkin [6] and extended to the true two dimensional case. The effect of switching from a single path coherent motion of vortices at transport currents just above the critical value $I_{c}$ to multiple paths and later to the "flux flow" state at higher currents is proposed to describe typical features in I-V characteristics of our samples.

\section{SAMPLE PREPARATION}

The investigated nanobridges have been structured in epitaxially grown (001)-oriented $\mathrm{YBa}_{2} \mathrm{Cu}_{3} \mathrm{O}_{7-\delta}$ films with thickness $\mathrm{d} \approx 50 \mathrm{~nm}$ on $(100) \mathrm{SrTiO}_{3}$ substrates. Films have been prepared by, both, laser ablation and off-axis rf magnetron sputtering techniques with no significant difference in quality. Film deposition processes as well as film properties have been discussed elsewhere [7],[8].

Single and trilayer electron beam resist lithography and plasma etching methods described in [1] have been used to define submicron bridges. Attempts to use chemical wet etching for nanoscale structuring of $\mathrm{YBaCuO}$ films proposed in [9] have been made but have not been successful. At the same time it has been found that wet etching is a very convenient tool to define structures with sizes above a few microns. The sizes of nanobridges have been estimated from SEM micrographs (Fig.1). Pictures of bridges have been made after electrical measurements have been performed to

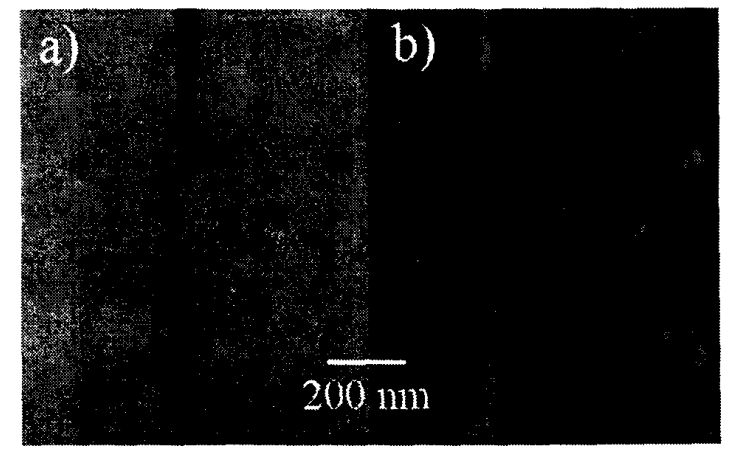

Fig. 1. SEM pictures of YBaCuO nanobridges with width $w=200 \mathrm{~nm}$ and lengths $l=70 \mathrm{~nm}$ (a) and $l=300 \mathrm{~nm}(\mathrm{~b})$. 
avoid destructive treatment of the superconducting film by the electron beam.

\section{RESULTS}

An example of nanobridges structured in an $\mathrm{YBaCuO}$ $50 \mathrm{~nm}$ thick film is shown in Fig.1. The critical temperature $T_{c}$ of the investigated bridges has been determined by a four point resistance measurement technique with applied current of $10 \mu \mathrm{A}$ and a critical temperature criterion $T_{c}=T\left(R=0.002 \cdot R^{100 K}\right)$. Some bridges have shown negligible reduction of $T_{c}$ in comparison with $T_{c}$ of unstructured films (for instance, $T_{c}=88 \mathrm{~K}$ for a $200 \mathrm{~nm}$ wide bridge). For other nanobridges of the same size significant degradation of $T_{c}$ down to $40 \mathrm{~K}$ has been detected. This can be explained in terms of oxygen loss through film defects during ion milling which heats the sample and significant spread in film quality from run to run. Critical current densities $j_{c}$ estimated in an assumption of a homogeneous current distribution along the bridge cross-section are up to $5 \cdot 10^{10} \mathrm{~A} / \mathrm{m}^{2}$ at $\mathrm{T}=4.2 \mathrm{~K}$ for those samples which shown no degradation of $T_{c}$ due to structuring. However, as has been shown in [10] for nanobridges of hyperbolic shape the supercurrent distribution inside the bridge is strongly inhomogeneous and depends on the angle of the neck of the bridge $\beta$.

Fig. 2 shows the measured values of the critical current $I_{c}$ as a function of the bridge width $w$. Only results for bridges with no reduction in $T_{c}$ are presented. The solid line has been calculated using the relation for the critical current from [10]:

$$
\mathrm{I}_{\mathrm{c}}=\mathrm{I}_{0} \frac{\beta}{\pi} \cdot \frac{a}{\delta} \sqrt{1-\left(\sin \beta-\frac{\delta}{a}\right)^{2}},
$$

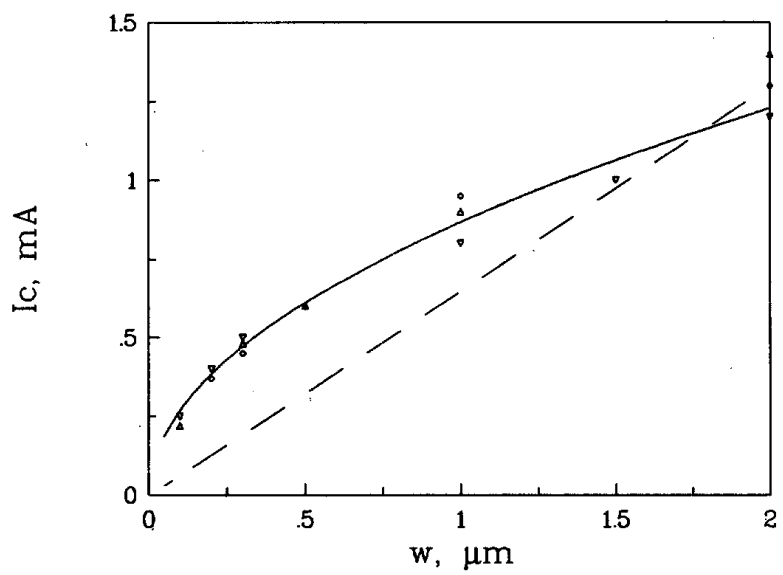

Fig.2. The critical current $I_{c}$ as a function of the width $w$ of a bridge at $\mathrm{T}=4.2 \mathrm{~K}$. The solid line is calculated using (1) and the dashed line is plotted assuming constant critical current density $\mathrm{j}_{\mathrm{c}}$ independent from the width of the bridge. derived for symmetric hyperbolic bridges with a neck angle $\beta$ and focal point $a=\mathrm{w} / 2 \sin \beta$ assuming that the critical current is determined by the edge pinning of vortices at the boundary of the bridge with the nucleation distance of a vortex from the edge of the bridge $\delta=4 \mathrm{~nm}$, angle $\beta=\pi / 2$ and model parameter $\mathrm{I}_{0}=0.1 \mathrm{~mA}$. Our experimental data do not fit to a straight line which has been plotted assuming the dominating role of bulk pinning of vortices in a bridge, i.e., assuming a constant supercurrent density $j_{c}$ for bridges of different width. From these results together with the high values of $j_{c}$ we infer the importance of edge pinning for our structures.

In Fig. 3 the experimental current-voltage I-V and the first derivative $\mathrm{dV} / \mathrm{dI}-\mathrm{I}$ characteristics of a nanobridge with $w=200 \mathrm{~nm}$ and $1 \approx 150 \mathrm{~nm}$ are presented. There are a few characteristic features to be seen in these curves: 1) quadratic voltage on current dependence $V \propto\left(I_{c}-I\right)^{2}$, which is typical for flux-flow type I-V characteristics; 2 ) steps in the I-V curve better resolved in the derivative curve. These steps are present in the I-V characteristics of all investigated nanobridges but the position of the steps may vary from sample to sample.

These steps can not be explained by the presence of a series of weak links in the bridge area. First, the lengths of our bridges are smaller or comparable with typical sizes of single crystal domains in the film, so there is no room for significant variation of film properties. Second, the steps are well reproducible from sample to sample, which does not correlate with random distribution of grains and grain boundaries in the film. Last, rather large amount of steps (up to 5) can usually be seen in the I-V characteristics.

An attempt to simulate the experimental I-V characteristics has been made. Our simulations have been performed in the framework of the Aslamazov-Larkin model [6] of viscous Abrikosov vortices motion based on the Ginzburg-Landau theory by numerically solving the equation of motion $F=\eta \mathbf{v}$

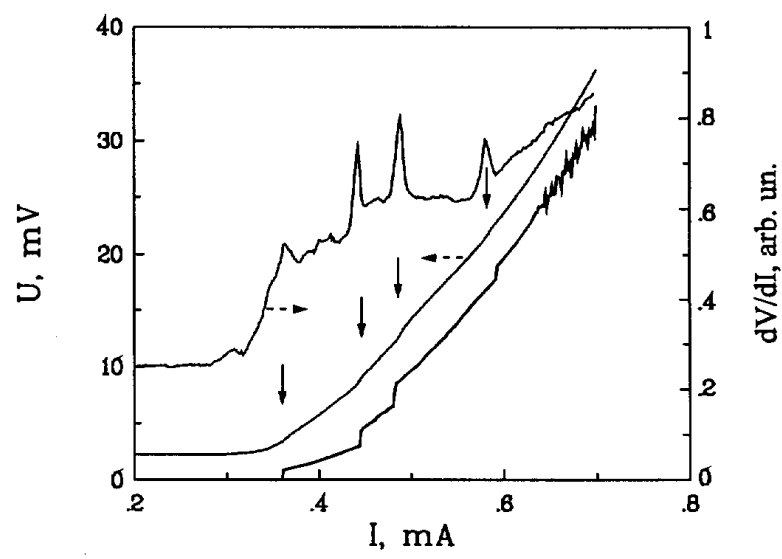

Fig.3. The V-I and dV/dI-I characteristics of $200 \mathrm{~nm}$ wide nanobridge at $\mathrm{T}=4.2 \mathrm{~K}$ (thin lines). The thick line is a simulation assuming viscous vortex motion (see text) 
for every vortex in a bridge. Here, $\mathbf{F}$ is the sum of forces acting on a vortex due to vortex-vortex, vortex-transport current and vortex-edge of a bridge interactions, $\eta$ is the viscosity coefficient of a moving vortex [11] and $v$ is the vortex velocity.

In contradiction to earlier simulations [1],[6],[10] which have been performed assuming a one-dimensional motion of vortices along the line of maximal transport current concentration our calculations have been made by deriving a two-dimensional equation of vortex motion and permitting for vortices to nucleate along bridge edges. To our knowledge, there are very few publications with twodimensional simulations of flux dynamics in finite structures in superconducting thin films [12]. An expression for the supercurrent distribution for nanobridges of hyperbolic shape with different angles of the bridge neck $\beta$ from [10] has been used.

The effect of recombination of vortices motion from single path along the narrowest part of a bridge at transport currents just above the critical value (in agreement with earlier onedimensional simulations) to double path at higher currents, later to multiple paths and finally to an incoherent flow state (Fig.4) has been found from simulations. Such switching in the vortex motion gives rise to steps in the corresponding simulated $\mathrm{I}-\mathrm{V}$ curves and an increase of the effective resistance of the bridge due to the sudden change in the amount of vortices crossing the bridge (Fig.3, thick line). To calculate the simulated curve in Fig. 3 the following parameters have been used: $w=200 \mathrm{~nm}, \delta=4 \mathrm{~nm}, \beta=\pi / 3$. Although the position of the steps in the experimental $\mathrm{I}-\mathrm{V}$ characteristics as well as the general shape of the curves can be fitted well by the simulated curves the amplitude of these steps is much smaller than that obtained from simulations. This fact can not be explained by thermal smearing and remains unclear.

The value of the viscous drag coefficient of vortex motion $\eta$ obtained from simulations is about $10^{-9} \mathrm{~kg} / \mathrm{m} \cdot \mathrm{sec}$ which is

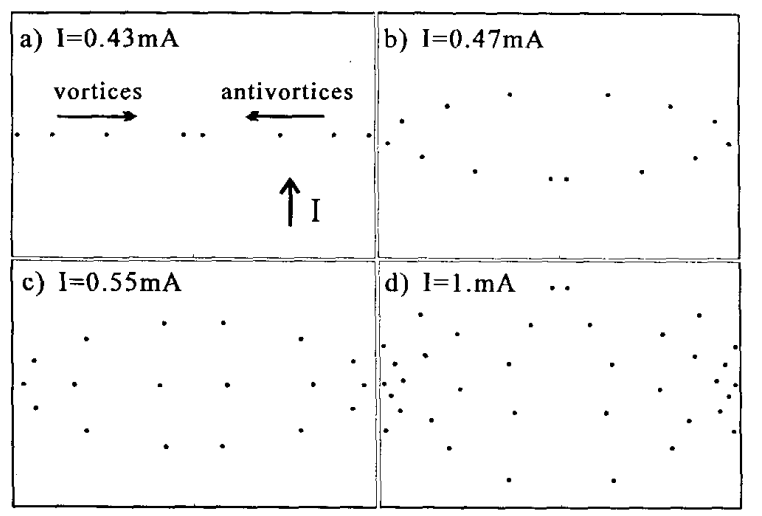

Fig.4. Snapshots of simulated motion of vortices inside the $200 \mathrm{~nm}$ wide hyperbolic nanobridge with $I_{c}=0.36 \mathrm{~mA}$ at different transport currents. approximately a factor 50 lower than the theoretical value [11]: $\eta=\Phi_{0}^{2} / 2 \pi \xi^{2} \rho_{\mathrm{n}}$, where $\rho_{\mathrm{n}}$ is the normal state resistivity of our structures $\left(\approx 10^{-6} \Omega \cdot \mathrm{m}\right)$. This reduced $\eta$ may stem from the fact that $\rho_{n}$ below $T_{c}$ is not well known. The average velocity of the vortex in a bridge is found to be in the order of $10^{5} \mathrm{~m} / \mathrm{sec}$.

Under microwave irradiation Shapiro-like steps appear in the I-V characteristics of our samples (Fig.5). The position of the steps is in agreement with the Josephson voltagefrequency relation $\mathrm{V}_{\mathrm{n}}=\mathrm{n} \Phi_{0} f(\mathrm{n}=0,1,2 \ldots)$, where $f$ is the frequency of applied microwave field. A decrease of the critical current with no further oscillations with increase of microwave irradiation power has been observed. This coincides with data obtained by other authors [1],[13] and can be explained on the basis of Abrikosov vortices motion synchronised by the external microwave field [14].

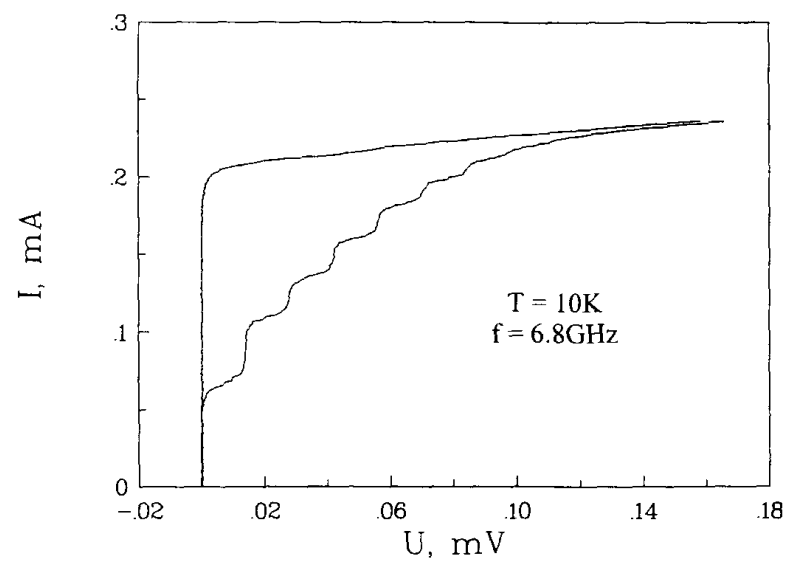

Fig.5. The I-V curves of a $100 \mathrm{~nm}$ wide nanobridge with (a) and without (b) an applied microwave field $(f=6.8 \mathrm{GHz})$ at $T=10 \mathrm{~K}$.

\section{CONCLUSION}

Fabrication of nanobridges with linear sizes of $\sim 100 \mathrm{~nm}$ with reproducible characteristics by e-beam lithography is well possible but strongly depends on the quality of the superconducting thin films.

The variation of the critical current with the width of the bridges indicates the dominating role of edge pinning of vortices. The idea of switching from a single path coherent motion of vortices at transport currents just above the critical value $I_{c}$ to multiply paths and later to an incoherent flow state at higher currents can be used to describe the typical features in the I-V characteristics. The viscous drag coefficient $\eta$ is found to be a factor of 50 lower than one estimated from the Bardeen-Stephen model. The effect of synchronisation of the vortex motion by external microwave field has been observed. 


\section{REFERENCES}

[1] M.J.M.E. de Nivelle, "Transport properties of nanobridges in high- $T_{c}$ superconducting YBaCuO films," Ph.D. thesis University of Twente, Enschede, the Netherlands, 1993.

[2] J.Schneider, H.Kohlstedt and R.Wördenweber, "Nanobridges of optimized $\mathrm{YBaCuO}$ thin films for superconducting fluxflow type devices," Appl.: Phys. Lett., vol.63 (17), pp.24262428, October 1993.

[3] K.K.Likharev, "Superconducting weak links," Review of Morden Physics, vol. 51, pp.101-159, January 1979.

[4] K.K.Likharev, "Vortex motion and the Josephson effect in superconducting thin bridges," Zh. Eksp. Teor. Fiz., vol. 61, pp.1700-1711, October 1971; Sov. Phys.-JETP, vol. 34, pp.906-912, April 1972.

[5] M.Mück and H.Rogalla, "Radiation from $\mathrm{Nb}_{3} \mathrm{Ge}$ nanobridges: Experiments and comparison with theory," Japanese Journal of Applied Physics, vol. 26. pp.1553-1554, 1987.

[6] L.G.Aslamazov and A.I. Larkin, "Josephson effect in wide superconducting bridges," Zh. Eksp. Teor. Fiz., vol. 68, pp.766-775, February 1975; Sov. Phys.-JETP, vol. 41, pp.381-396, 1975.

[7] J.Gao, B.Häuser and H.Rogalla, "High critical current density ultrathin $\mathrm{YBaCuO}$ films made by modified rf-magnetron sputtering technique," J. Appl. Phys., vol. 67(5), pp.2512$2515,1990$.

[8] D.H.A.Blank, D.J. Adelerhof, J.Flokstra and H.Rogalla, "Preparation of YBaCuO thin films on various substrates by laser ablation," Physica C, vol.167, pp.423-432, 1990.

[9] C.I.H.Ashby, J.Martens et al., "Improved aqueous etchant for high $T_{c}$ superconductor materials," Appl.Phys.Lett., vol.60 (17), pp.2147-2149, April 1992.

[10] H.Rogalla, "High $T_{c}$ Josephson contacts. Preparation and properties," Habilitation thesis, University of Giessen, Germany, 1986.

[11] J.Bardeen and M.J.Stephen, "Theory of the motion of vortices in superconductors," Phys. Rev., vol.140, p.A1197, 1965.

[12] M.Machida and H.Kaburaki, "Direct simulation of the timedependent Ginzburg-Landau equation for type-II superconducting thin films: vortex dynamics and V-I characteristics," Phys. Rev. Lett., vol.71 (19), pp.3206-3209, November 1993.

[13] M.A.M.Gijs and R.J.E.Jansen "Microwave response of YBaCuO thin-film Dayem bridges," Appl. Phys. Lett., vol.56 (15), pp. 1484-86, April 1990.

[14] A.T.Golovashkin and A.N.Lykov "Investigation of bridge junctions made of high-temperature superconductor $\mathrm{Nb}_{3} \mathrm{Sn}$," Zh. Eksp. Teor. Fiz., vol. 74, p.214, 1978; Sov. Phys.-JETP, vol. 47, pp.110-115, January 1978 . 